\title{
O. NOTATION AND CONVENTION
}

0.1. A topological space is called nice if it is Hausdorff, locally compact, paracompact and with a countable basis for its topology.

0.2. If $A$ is a topological space and $X \subset A$, then $\mathrm{cl}_{A}(X)$ (resp. int $A(X)$ ) denotes the closure (resp. interior) of $X$ in $A$.

0.3. If $A$ and $B$ are topological spaces, then $A \sqcup B$ denotes their disjoint union.

0.4. For any set $A, 1_{A}$ (or id ${ }_{A}$ ) denotes the identity map of $A$.

0.5. Smooth means always differentiable of class $C^{\infty}$.

0.6. The connected components of a smooth manifold may have different dimensions. Given a smooth manifold $M$ we denote by $T M$ its tangent bundle. If $x \in M, T M_{x}$ denotes the tangent space of $M$ at $x$. If $N$ is another smooth manifold and $f: M \rightarrow N$ is smooth, $d f: T M \rightarrow T N$ denotes the differential of $f$; if $x \in M$, then $d f_{x}: T_{x} \rightarrow_{T N}(x)$ denotes the restriction of $d f$. The smooth map $f$ is called submersive if $d f$ is surjective for any $x \in M$. 0.7 . Let $A$ be a topological space and let $X, Y$ and $Z$ be subsets of $A$. Let $f$ and $g$ be maps defined on $X$ and $Y$ respectively. We say that $f$ equals $g$ near $Z$ (denoted $f=g$ near $Z$ ) if there exists a neighborhood $U$ of $Z$ such that $f|X \cap U=g| Y \cap U$. The same terminology is also used in other similar situations (for example $X=Y$ near $Z$ means that there exists a neighborhood $U$ of $Z$ such that $X \cap U=Y \cap U)$ ).

0.8. $R$ denotes the field of real numbers; $R_{+}=\{r \in R ; r \geq 0\}$ and $R_{+}^{*}=\{r \in R ; r>0\}$. 